\title{
ANÁLISE DE CARACTERÍSTICAS REPRODUTIVAS INDICADORAS DE PUBERDADE EM TOURINHOS PANTANEIRO
}

\author{
ANALYSIS OF REPRODUCTIVE CHARACTERISTICS INDICATORS OF PUBERTY \\ IN YOUNG PANTANEIRO BULLS
}

\author{
Juliano, R.S. ${ }^{1}$, Ramos, A.F..$^{*}$, Santos, S.A. ${ }^{1}$ e Abreu, U.G.P. de ${ }^{1}$ \\ ${ }^{1}$ Embrapa Pantanal. CP 109. CEP 79320-900. Corumbá-MS. Brazil. *raquel@cpap.embrapa.br \\ ${ }^{2}$ Embrapa Recurso Genético e Biotecnologia. Brasília-DF. Brazil.
}

\section{PALAVRAS CHAVE ADICIONAIS}

Gado de corte. Conservação in situ.

\section{RESUMO}

Descendentes de animais oriundos da Península Ibérica, os bovinos Pantaneiros foram introduzidos no Brasil na época do descobrimento. Características reprodutivas foram monitoradas em dez tourinhos, no intervalo de idade de dez a vinte meses, criados em pastagens nas condições de Brasil central com objetivo de determinar a idade à puberdade. Mensalmente eram mensuradas a circunferência, o comprimento e a largura testicular. O sêmen foi coletado e os aspectos de morfologia e físicos do ejaculado avaliado. Foi avaliada a idade do desprendimento entre pênis e prepúcio, do aparecimento da primeira célula espermática, da primeira célula espermática móvel e da puberdade seminal.

\section{SUMMARY}

The Pantaneiro cattle are descendant from animals brought by settlers in the beginning of the colonization of Brazil. Ten months old Pantaneiro steers, raised under pasture conditions in Brazil, were used to study puberty. Monhtly measurement of scrotal circumference and testicular width and length were recorded. Semen was collected by eletroejaculation and evaluated according to physical and morphological aspects. Penile and prepuce detachment percentages were also evaluated. The ages of first spermatozoa presence in the ejaculate, first motile spermatozoa in the ejaculate, seminal puberty and detachment between penis and prepuce was studied.

Presentado al Congreso SERGA (2010, Asturias).
AdDitional KeYWORDS

Beef cattle puberty. In situ conservation.

\section{INTRODUÇÃO}

Os bovinos Pantaneiros são animais trazidos da Península Ibérica na época do descobrimento das Américas. Ao longo destes três séculos passaram por um forte processo de seleção natural, com o qual adquiriram maior adaptabilidade às condições edafoclimáticas do Pantanal até sua progressiva substituição com outras raças de corte, como nos sistemas de cruzamento que vem sendo desenvolvidos no Brasil citado por Souza et al. (2010). O bovino Pantaneiro é descendente de raças espanholas introduzidas na América durante o processo de colonização da bacia do rio da Prata. Mais de três séculos de adaptação às pastagens nativas e regiões alagáveis do Pantanal conferiram a ele rusticidade, prolificidade e habilidade para sobreviver em condições de estresse térmico, hídrico e alimentar, que afetam diretamente o sistema os índices reprodutivos (Correa-Calderón et al., 2009). Em 1984 foi implantado o núcleo de conservação in situ para caracterização do Bovino Pantaneiro na fazenda Nhumirim, pertencente a Embrapa Pantanal, e desde então o rebanho vem sendo caracterizado em seus aspectos genéticos e produtivos (Abreu et al., 2000).

A idade à puberdade (IP) é uma característica importante para o sistema extensivo de produção do Pantanal, que se baseia 
principalmente na cria de bezerros. Goddard (2009) define características de adaptação como as necessárias para sobrevivência e desempenho reprodutivo dos animais. A IP neste contexto é uma importante variável indicadora de capacidade de adaptação (adaptedness) dos bovinos. O objetivo deste trabalho foi caracterizar o desenvolvimento puberal de reprodutores bovinos do grupamento genético Pantaneiro através de avaliações morfométricas e seminais.

\section{MATERIALE MÉTODOS}

Dez reprodutores do grupamento genético Pantaneiro foram transferidos da Fazenda Nhumirim da Embrapa Pantanal, Corumbá-MS, para o Campo Experimental Sucupira da Embrapa Recursos Genéticos e Biotecnologia, Brasília-DF, Brasil, aos dez meses de idade. Os animais permaneceram em pastagem de Brachiaria brizanta e mineralizados, sendo submetidos mensalmente à avaliação andrológica dos dez aos vinte meses para a caracterização cronológica dos eventos que concernem à puberdade. As variáveis acompanhadas foram idade (Id), peso corporal (PC), circunferência torácica (CT), escore de desprendimento do prepúcio (EDP), circunferência testicular (CTe), comprimento testicular (Comp), largura testicular (LT), espessura testicular(ET) e volume testicular (VT). Foi considerada IP quando os animais apresentaram o mínimo de 50 milhões de espermatozóides no ejaculado total com pelo menos 10\% de motilidade (Wolf et al., 1965). Para isso, os reprodutores tiveram o sêmen colhido por eletro-ejaculador e seu ejaculado avaliado por microscopia de luz.

A variável resposta caracterizou resposta binomial, o tourinho era considerado púbere (1) ou não (0) a cada avaliação. Os dados coletados mensalmente de cada tourinho foram considerados dados repetidos no tempo de resposta binária. E foram analisados por meio de estimação de equações lineares generalizadas (EEG) e função de ligação logística, utilizando o procedure GENMOD do pacote estatístico SAS.

\section{RESULTADOSEDISCUSSÃO}

A idade média a puberdade para os 10 tourinhos avaliados foi estimada em 17,20 \pm 0,786 meses. O intervalo da amplitude da característica foi encontrado de 13 a 20 meses de idade. As variáveis contextuais Id, EDP e Comp foram observados efeitos significativos para a IP. Na tabela I são apresentadas as estimativas dos efeitos significativos.

No Brasil, Castro et al. (1989) observaram espermatozóides no ejaculado de tourinhos Nelore, criados exclusivamente a pasto, aos 12-14 meses de idade. Por outro lado, Unanian (1997), observou na mesma raça, que o aparecimento da puberdade em machos Nelore variando de 12,2 a 16,0 meses de idade.

A média e a amplitude da IP observada para os bovinos Pantaneiros são semelhantes ao encontrado na literatura para a raça Nelore no Brasil. As pesquisas realizadas no Brasil, considerando os aspectos relacionados a puberdade, salientam a maior

Tabela I. Estimativas das variáveis contextuais significativas para idade à puberdade. (Estimate of significant contextual variables for puberty age).

\begin{tabular}{lcc}
\hline Variável & Estimativa & Erro padrão \\
\hline Intercepto & $-8,796$ & 3,064 \\
Idade (Id) & 0,992 & 0,197 \\
EDP 0 & $-0,659$ & 0,967 \\
EDP 1 & $-1,605$ & 1,849 \\
EDP 2 & $-1,471$ & 1,729 \\
EDP 3 & 1,556 & 0,638 \\
EDP 4 & $-1,054$ & 0,803 \\
EDP 5 & 0 & 0 \\
Comprimento testicular & $-0,806$ & 0,244 \\
\hline
\end{tabular}

EDP Escore de desprendimento do prepúcio. 
dependência de seu aparecimento do estado corporal do animal do que da idade. Entretanto na análise com o bovino Pantaneiro a variável Id foi significativa, provavelmente em função do pequeno tamanho corporal dos animais, pois sendo animais de tamanho pequeno (Abreu et al., 2004) a exigência nutricional é menor o que facilita a adaptação ao ambiente do Pantanal em que ocorre períodos cíclicos críticos de alimentação para bovinos em pastagens nativas.

Nogueira (2004) cita que a puberdade nas raças zebuínas na América do Sul são tardias, e uma das estratégias para torná-las mais precoce pode ser a utilização de raças taurinas, como o bovino Pantaneiro, em sistema de cruzamento. $\mathrm{O}$ formato testicular do bovino Pantaneiro especialmente o comprimento foi a característica morfológica testicular com efeito significativo positivo na IP, e deverá ser objeto de futuras pesquisas. Unanian et al. (2000) observaram que em função da forma mais alongada dos testículos de zebuínos, e recomendaram que, ao se praticar seleção em animais jovens, deve-se utilizar o formato testicular para avaliar com maior precisão sobre o potencial do futuro reprodutor.

A idade média do desprendimento entre o pênis e o prepúcio observado para os EDP 1, EDP 2, EDP 3, e EDP 4 e EDP 5 foram 12,85 meses, 13,22 meses, 14,47 meses, 14,9 meses e 16,39 meses, respectivamente. Freneau et al. (2006) na raça Nelore observaram a idade de 18,10 meses. E ressaltaram que apesar de o desprendimento total entre as mucosas ter sido observado em todos os animais é possível que essa característica seja determinada em menor idade, pois nas colheitas de ejaculado, a exposição do pênis não ocorreu em todos os animais.

As equações probabilísticas estimadas a partir do modelo de regressão logística com dado repetido no tempo e distribuição binomial são apresentadas na figura $\mathbf{1}$.

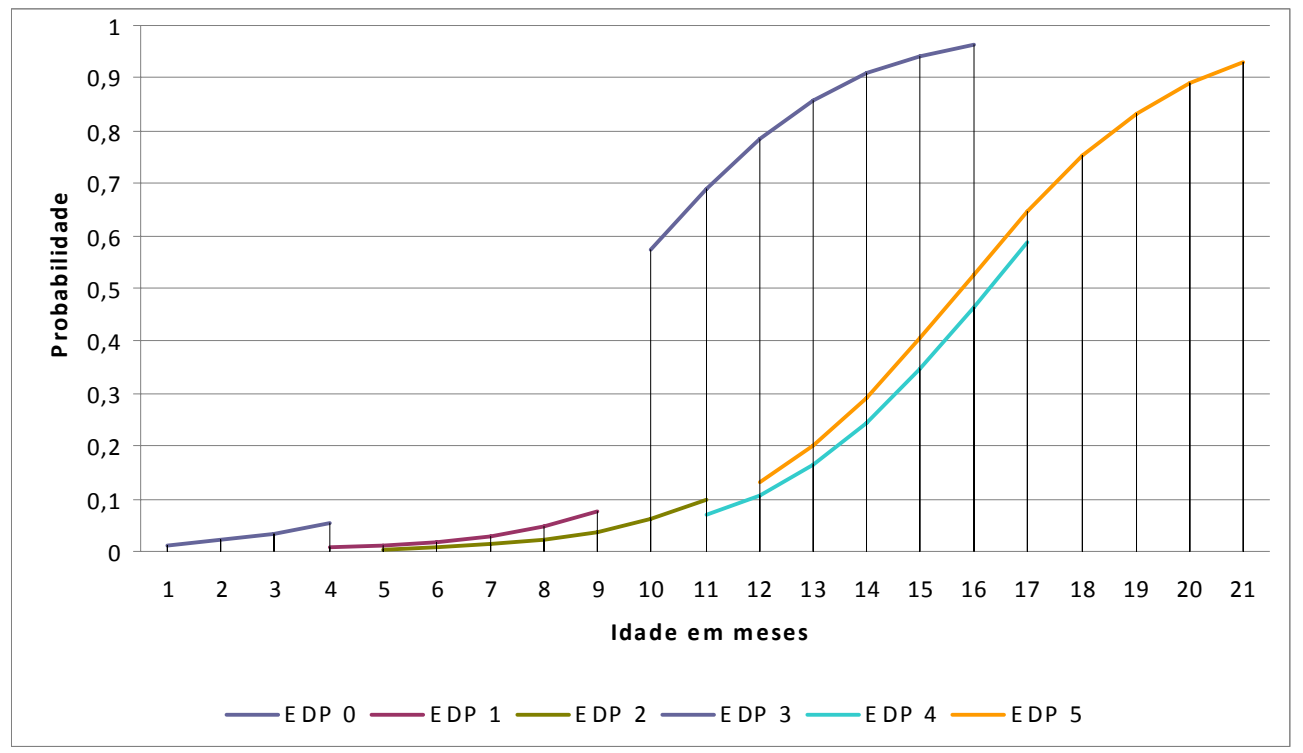

Figura 1. Curvas de probabilidade de atingir a puberdade em função da idade e do escore de desprendimento entre o pênis e o prepúcio. (Probability curves to reach puberty depending on age, penis and prepuce detachment). 


\section{JULIANO, RAMOS, SANTOSE ABREU}

Curvas de probabilidade foram estimadas para ocorrência de puberdade em função das características. As estimativas dos contrastes entre os EDP (escores de 0: prepúcio aderido a pele a 5 : prepúcio totalmente solto da pele) foram comparados, o contraste significativo foi entre os EDPs 3 e 4, os animais de escore 4 tiveram 13,60 vezes mais chance de serem púberes em relação aos de escore 3. O desprendimento entre o pênis e o prepúcio foi variável importante para determinação da puberdade dos bovinos Pantaneiros. Entretanto a grande maioria dos animais quando alcançavam o EDP 3 apresentavam as características de puberdade com a idade média de 14,47 meses.

\section{BIBLIOGRAFIA}

Abreu, U.G.P., Cobuci, J.A., Silva, M.V.G.B. da y Sereno, J.R.B. 2004. Uso de modelos no lineales para ajuste de la curva de crecimento de bovinos Pantaneiros. Arch. Zootec., 53: 367-370.

Abreu, U G. P., Sereno, J.R.B. y Lara, M.A.C. 2000. Evaluación zootécnica del núcleo de conservación in situ del bovino Pantaneiro en el Pantanal Brasileño. Arch. Zootec., 49: 27-30.

Castro, V.M., Vale Filho, V.R., Reis, S.R. e Oliveira, H.N. 1989. Puberdade e início de maturação sexual em touros Nelore, de 10-14 meses de idade. Rev. Bras. Reprod. Anim. (Supl. vol. 1): 183.

Correa-Calderón, A., des Santos, G., Avendaño, L., Rivera, F., Álvarez, D., Ardón, F., Díaz, R. y Collier, R. 2009. Enfriamento artificial y tasa de concepción de vaquillas Holstein com estrés térmico. Arch. Zootec., 58: 231-239.

Freneau, G.E., Vale Filho, V.R., Marques Jr, A.P e Maria, W.S. 2006. Puberdade em touros Nelore criados em pasto no Brasil: características corporais, testiculares e seminais e de índice de capacidade andrológica por pontos. Arq. Bras. Med. Vet. Zootec., 58: 1107-1115.

Goddard, M. 2009. Fitness traits in animal breeding
Idade mais precoce ao observado para a raça Nelore por Unanian (1997) e Freneau et al. (2006).

\section{CONCLUSÕES}

A puberdade observada no bovino Pantaneiro é semelhante à principal raça de corte do Brasil. O desprendimento do pênis do prepúcio é característica importante na determinação da puberdade dos bovinos Pantaneiros, sendo necessária mais pesquisa para melhor inferir sobre a importância do formato dos testículos e a correlação com características de adaptação a região do Pantanal.

programs. In: Van der Werf, J., Graser, H-U., Frankham, R., Gondro, C. (Eds). Adaptation and fitness in animal populations. Springer. New York. pp. 41-52.

Nogueira, G.P. 2004. Puberty in South American Bos indicus (zebu) cattle. Anim. Reprod. Sci., 82-83: 361-372.

Souza, J.C., Perotto, D., Abrahão, J.J, Freitas, J.A., Ferraz Filho, P.B., Weaber, R.L. e Lamberson, W.R. 2010. Estimativa das distâncias genéticas e componentes principais em bovinos de corte no Brasil. Arch. Zootec., 59: 480-485.

Unanian, M.M., Silva, A.M.D.F., McManus, C. e Cardoso, E.P. 2000. Características biométricas testiculares para avaliação de touros zebuínos da raça Nelore. Rev. Bras. Zootecn., 29: 136144.

Unanian, M.M. 1997. A procura de marcadores de precocidade em gado Nelore. In: O Nelore do Século XXI, 4, ABCZ. Uberaba. Minas Gerais. Anais... Uberaba-MG. pp. 51-57.

Wolf, F.R., Almquist, J.O. and Hale, E.B. 1965. Prepuberal behavior and pubertal characteristics of beef bulls on high nutrient allowance. J. Anim. Sci., 24: 761-764. 